\title{
How to construct a coordinate representation of a Hamiltonian operator on a torus
}

\author{
SUMIO ISHIKAWA* TADASHI MIYAZAKI, \\ KAZUYOSHI YAMAMOTO ${ }^{\dagger}$ and MOTOWO YAMANOBE ${ }^{\ddagger}$ \\ Department of Physics, Science University of Tokyo, \\ Kagurazaka, Shinjuku-ku, Tokyo 162, Japan
}

October 1995

PACS 03.65.-w - Quantum mechanics

\begin{abstract}
The dynamical system of a point particle constrained on a torus is quantized $\grave{a}$ la Dirac with two kinds of coordinate systems respectively; the Cartesian and toric coordinate systems. In the Cartesian coordinate system, it is difficult to express momentum operators in coordinate representation owing to the complication in structure of the commutation relations between canonical variables. In the toric coordinate system, the commutation relations have a simple form and their solutions in coordinate representation are easily obtained with, furthermore, two quantum Hamiltonians turning up. A problem comes out when the coordinate system is transformed, after quantization, from the Cartesian to the toric coordinate system.
\end{abstract}

\footnotetext{
*E-mail: liszt@grad.ap.kagu.sut.ac.jp

$\dagger^{\dagger}$ E-mail: ymt@grad.ap.kagu.sut.ac.jp

${ }^{\ddagger}$ E-mail: yamanobe@grad.ap.kagu.sut.ac.jp
} 


\section{Introduction}

How can one formulate, in the Hamiltonian formalism, the classical system of a particle constrained on a curved surface? Starting with a classical-mechanical system for that constrained particle, how can one obtain a quantum-mechanical one?

The first question is solved in use of the Dirac formalism to the system[四]. In the Hamiltonian formalism, Poisson brackets play a main role when we deal with a system without constraints. When we are confronted, on the other hand, with a constrained system, the Dirac brackets take their place. Making use of the Dirac brackets, we can consistently formulate the constrained system in an elegant way.

Noticing the point that the Dirac brackets for the constrained system play the role of the Poisson brackets for the unconstrained system, we can quantize the classical constrained system, i.e., we can answer the second question. The Dirac brackets are to be replaced, in quantization, by the commutators $(\times 1 / i \hbar)$. Along this canonical-quantization method, therefore, it is important that we describe the system in the language of the Hamiltonian formalism, not of the Lagrangian formalism.

Now, let us focus our attention on the system of a particle constrained on a two-dimensional closed surface. The simplest closed surface is a sphere. Quantum mechanics for a particle on a sphere has been studied by many researchers[2], among whom are Falck and Hirshfeld[3].

From the mathematical viewpoint, any compact orientable surface is known to be homeomorphic to a sphere or a connected sum of tori[四]. However, the quantum mechanics on a torus has not, within the knowledge of the present authors, been constructed, so we will work that out in the present paper. We are thus to have quantum mechanics on all compact orientable surfaces.

The present paper is organized as follows. In Sec.2 we study the classical system of a particle on a torus à la Dirac. Two coordinate systems are used, one is Cartesian and the other toric. The reason why the toric coordinate system is useful is that one can here easily obtain coordinate representations of momentum operators. In Sec.3 we quantize the system with the 
canonical-quantization method. In the classical theory of a particle constrained on a surface, the Dirac brackets of the canonical variables are complicated in structure if one deals with the system in Cartesian coordinates, as shown in Sec.2.1. Therefore, it is difficult to obtain the coordinate representations of momentum operators. There are two ways available to avoid that difficulty. One is to choose a suitable coordinate system for the dynamical system in which the Dirac brackets come to have simple structures. The other was shown by Homma, Inamoto and Miyazaki in case of a sphere[0]. They took up the time derivative of the equation of a surface as a constraint condition. We go along the former way in the present paper. In Sec.3.1 we study quantization in toric coordinates. Two Hamiltonian operators are shown to exist; one consists only of differential terms, and the other both of differential and functional ones. In Sec.3.2 we first quantize the constrained system in Cartesian coordinates, and transform the coordinates to the toric afterwards. It is found that the momentum operator becomes hermitian or not, depending on the stage at which we impose the constraint condition on the system. The last section is devoted to the conclusion and discussion of our analysis.

\section{Classical mechanics on a torus}

In $\mathbf{R}^{3}\left(x_{1}, x_{2}, x_{3}\right)$ we consider a particle on a torus which is generated by rotating a circle (radius: $a$, center: $(R, 0,0)$, in $x_{1}-x_{3}$ plane) about the $x_{3}$-axis. It is expressed by

$$
x_{3}^{2}-a^{2}+\left(\sqrt{x_{1}^{2}+x_{2}^{2}}-R\right)^{2}=0
$$

\subsection{Classical mechanics in Cartesian coordinates}

The Lagrangian $L$ of this system is given by

$$
L=\frac{1}{2} m \dot{x}_{i} \dot{x}_{i}-x_{4}\left(x_{3}^{2}-a^{2}+\left(\sqrt{x_{1}^{2}+x_{2}^{2}}-R\right)^{2}\right) .
$$

where $x_{4}(t)$ is a Lagrange multiplier and treated as an independent variable, and $m$ is the mass of the particle. Here and henceforth the summation convention of the repeated indices is 
employed. From Eq.(2.2), we obtain canonical momenta:

$$
\begin{gathered}
P_{i} \equiv \frac{\partial L}{\partial \dot{x}_{i}}=m \dot{x}_{i}, \quad(i=1,2,3), \\
P_{4} \equiv \frac{\partial L}{\partial \dot{x}_{4}} \equiv \phi_{1} \approx 0
\end{gathered}
$$

where " $\approx$ " means weak equality in Dirac's sense. Equation (2.4) is the primary constraint of this system. From Eqs.(2.2) (2.4), the Hamiltonian $H$ is given by

$$
H=\frac{1}{2 m} P_{i} P_{i}+x_{4}\left[x_{3}^{2}-a^{2}+\left(\sqrt{x_{1}^{2}+x_{2}^{2}}-R\right)^{2}\right]+u P_{4},
$$

where $u\left(\equiv \dot{x_{4}}\right)$ is a Lagrange multiplier. To keep consistency for the system, all constraints are to be imposed after working out all Poisson brackets. In order that the system be compatible with the dynamical evolution, we require all constraints to be conserved throughout all time. This requirement is called a consistency condition. In our case, it is necessary that $\phi_{2} \equiv \dot{\phi}_{1} \approx 0$. We have thus a new constraint (secondary constraint) $\phi_{2} \approx 0$ on the system. Furthermore, we impose the consistency condition on $\phi_{2} \approx 0$. In general, above arguments continue till either no new constraint turns up further or a condition on a Lagrange multiplier in the Hamiltonian is obtained. In this paper, the consistency condition finally gives a condition on $u$ in Eq.(2.5).

$$
\begin{aligned}
\dot{\phi}_{1} \approx & x_{3}^{2}-a^{2}+\left(\sqrt{x_{1}^{2}+x_{2}^{2}}-R\right)^{2} \equiv \phi_{2} \approx 0, \\
\dot{\phi}_{2} \approx & x_{i} P_{i}-\frac{R}{\sqrt{x_{1}^{2}+x_{2}^{2}}}\left(x_{1} P_{1}+x_{2} P_{2}\right) \equiv \phi_{3} \approx 0, \\
\dot{\phi}_{3} \approx & \frac{1}{m \sqrt{x_{1}^{2}+x_{2}^{2}}}\left[P_{i} P_{i}+\frac{R\left(x_{1} P_{1}+x_{2} P_{2}\right)^{2}}{x_{1}^{2}+x_{2}^{2}}-R\left(P_{1}^{2}+P_{2}^{2}\right)\right] \\
& +2 x_{4}\left[x_{3}^{2}+\left(\sqrt{x_{1}^{2}+x_{2}^{2}}-R\right)^{2}\right] \equiv \frac{\phi_{4}}{m} \approx 0, \\
\dot{\phi}_{4} \approx & -\frac{3}{m R} \frac{x_{i} P_{i}}{\sqrt{x_{1}^{2}+x_{2}^{2}}}\left(2 x_{4} a^{2}-\frac{P_{j} P_{j}}{m}\right)-2 u a^{2} \approx 0 .
\end{aligned}
$$

Equation (2.9) determines the Lagrange multiplier $u$ in the Hamiltonian (2.5). Note that all constraints are found to be second-class; i.e. there is no constraint which has zero Poisson brackets with all other constraints. Therefore, Eq. 2.5) is rewritten as

$$
H=\frac{1}{2 m} P_{i} P_{i}+x_{4} \phi_{2}-\frac{3}{2 m R a^{2}} \frac{x_{i} P_{i}}{\sqrt{x_{1}^{2}+x_{2}^{2}}}\left(2 x_{4} a^{2}-\frac{P_{j} P_{j}}{m}\right) \phi_{1} .
$$


At this stage the Poisson bracket is defined by

$$
\left.\{A, B\}_{\mathrm{P}} \equiv \frac{\partial A}{\partial x_{\mu}} \frac{\partial B}{\partial P_{\mu}}-\frac{\partial A}{\partial P_{\mu}} \frac{\partial B}{\partial x_{\mu}}, \quad \text { (summation over } \mu=1,2,3,4\right) .
$$

Equations (2.10) and (2.11) have a variable $x_{4}$ in themselves. We try to express $x_{4}$ in terms of other variables. From $\phi_{2} \approx 0$ and $\phi_{4} \approx 0$, we obtain

$$
x_{4} \approx \frac{1}{2 m a^{2}}\left[P_{i} P_{i}+\frac{R}{\sqrt{x_{1}^{2}+x_{2}^{2}}}\left(\frac{\left(x_{1} P_{1}+x_{2} P_{2}\right)^{2}}{x_{1}^{2}+x_{2}^{2}}-P_{1}^{2}-P_{2}^{2}\right)\right] .
$$

With this equation as well as Eq.(2.4), we regard the pair $\left(x_{4}, P_{4}\right)$ as dependent variables, and hence, the remaining constraints are $\phi_{2} \approx 0$ and $\phi_{3} \approx 0$.

Now, we introduce the Dirac bracket defined by

$$
\{F, G\}_{\mathrm{D}} \equiv\{F, G\}_{\mathrm{P}}-\left\{F, \phi_{\alpha}\right\}_{\mathrm{P}} C_{\alpha \beta}^{-1}\left\{\phi_{\beta}, G\right\}_{\mathrm{P}}
$$

with

$$
C_{\alpha \beta} \equiv\left\{\phi_{\alpha}, \phi_{\beta}\right\}_{\mathrm{P}}, \quad C_{\alpha \beta} C_{\beta \gamma}^{-1}=\delta_{\alpha \gamma}, \quad(\alpha, \beta, \gamma=1,2)
$$

From Eq. 2.13), the Dirac brackets of canonical variables are given as follows:

$$
\begin{aligned}
\left\{x_{i}, x_{j}\right\}_{\mathrm{D}}= & 0 \\
\left\{x_{i}, P_{j}\right\}_{\mathrm{D}}= & \delta_{i j}-\frac{1}{a^{2}}\left(x_{i}-\frac{x_{1} \delta_{1 i}+x_{2} \delta_{2 i}}{\sqrt{x_{1}^{2}+x_{2}^{2}}} R\right)\left(x_{j}-\frac{x_{1} \delta_{1 j}+x_{2} \delta_{2 j}}{\sqrt{x_{1}^{2}+x_{2}^{2}}} R\right), \\
\left\{P_{i}, P_{j}\right\}_{\mathrm{D}}= & -\frac{1}{a^{2}}\left[\left(x_{i}-\frac{x_{1} \delta_{1 i}+x_{2} \delta_{2 i}}{\sqrt{x_{1}^{2}+x_{2}^{2}}} R\right)\right. \\
& \times\left(P_{j}-\frac{R}{\sqrt{x_{1}^{2}+x_{2}^{2}}}\left(P_{1} \delta_{1 j}+P_{2} \delta_{2 j}-\frac{x_{1} P_{1}+x_{2} P_{2}}{x_{1}^{2}+x_{2}^{2}}\left(x_{1} \delta_{1 j}+x_{2} \delta_{2 j}\right)\right)\right) \\
& -\left(x_{j}-\frac{x_{1} \delta_{1 j}+x_{2} \delta_{2 j}}{\sqrt{x_{1}^{2}+x_{2}^{2}}} R\right) \\
& \left.\times\left(P_{i}-\frac{R}{\sqrt{x_{1}^{2}+x_{2}^{2}}}\left(P_{1} \delta_{1 i}+P_{2} \delta_{2 i}-\frac{x_{1} P_{1}+x_{2} P_{2}}{x_{1}^{2}+x_{2}^{2}}\left(x_{1} \delta_{1 i}+x_{2} \delta_{2 i}\right)\right)\right)\right] .
\end{aligned}
$$

Making use of the Dirac brackets, let us replace the weak equality " $\approx$ " by the strong equality "=". Finally the Hamiltonian is reduced to

$$
H_{E}=\frac{1}{2 m} P_{i} P_{i}, \quad(i=1,2,3) .
$$


Hamilton's equations of motion are

$$
\begin{aligned}
& \dot{x}_{i}=\left\{x_{i}, H_{E}\right\}_{\mathrm{D}}=\frac{P_{i}}{m}, \\
& \dot{P}_{i}=\left\{P_{i}, H_{E}\right\}_{\mathrm{D}}=-2 \alpha\left(x_{i}-\frac{x_{1} \delta_{1 i}+x_{2} \delta_{2 i}}{\sqrt{x_{1}^{2}+x_{2}^{2}}} R\right),
\end{aligned}
$$

where

$$
\alpha \equiv \frac{1}{2 m a^{2}}\left[P_{i} P_{i}+\frac{R}{\sqrt{x_{1}^{2}+x_{2}^{2}}}\left(\frac{\left(x_{1} P_{1}+x_{2} P_{2}\right)^{2}}{x_{1}^{2}+x_{2}^{2}}-P_{1}^{2}-P_{2}^{2}\right)\right] .
$$

In particular, as $R \rightarrow 0$ in Eqs.(2.19) and (2.20), we obtain

$$
\dot{P}_{i}=-\frac{1}{m a^{2}} P_{j} P_{j} x_{i} \equiv-m \omega^{2} x_{i}, \quad \omega: \text { const. }
$$

Equation (2.21) expresses an equation of circular motion, as was expected, with angular frequency $\omega=\sqrt{P_{j} P_{j}} /$ ma.

Now we can quantize the system by replacing Dirac brackets $(\times i \hbar)$ by commutators. However, since the Dirac brackets of the canonical variables are complicated in structure as shown in Eqs.(2.15) and (2.16), it is very difficult to obtain the coordinate representation of momentum operators. We will make no further mention of this difficulty in this section. In the next subsection, we define a toric coordinate system and consider the system in that coordinates.

\subsection{Classical mechanics in toric coordinates}

We introduce a new coordinate system and call it a toric one. The variables are defined as illustrated in Fig.1. The relationship between Cartesian coordinates and toric is given by

$$
\begin{aligned}
& x_{1}=(R+r \sin \theta) \cos \phi, \\
& x_{2}=(R+r \sin \theta) \sin \phi, \\
& x_{3}=r \cos \theta,
\end{aligned}
$$

with

$$
r \sin \theta>-R
$$


The Lagrangian $L$ in the toric coordinate system is

$$
L=\frac{1}{2} m\left[\dot{r}^{2}+r^{2} \dot{\theta}^{2}+(R+r \sin \theta)^{2} \dot{\phi}^{2}\right]-q_{4}(r-a)
$$

where $q_{4}$ is a Lagrange multiplier. Canonical momenta are given by

$$
\begin{aligned}
\Pi_{r} & \equiv \frac{\partial L}{\partial \dot{r}}=m \dot{r} \\
\Pi_{\theta} & \equiv \frac{\partial L}{\partial \dot{\theta}}=m r^{2} \dot{\theta} \\
\Pi_{\phi} & \equiv \frac{\partial L}{\partial \dot{\phi}}=m(R+r \sin \theta) \dot{\phi} \\
\Pi_{4} & \equiv \frac{\partial L}{\partial \dot{q}_{4}} \equiv \chi_{1} \approx 0 .
\end{aligned}
$$

Equation (2.27) is the primary constraint of this system. By the Legendre transformation, the Hamiltonian $H$ is obtained:

$$
H=\frac{1}{2 m}\left[\Pi_{r}^{2}+\frac{\Pi_{\theta}^{2}}{r^{2}}+\frac{\Pi_{\phi}^{2}}{(R+r \sin \theta)^{2}}\right]+q_{4}(r-a)+u \chi_{1},
$$

where $u\left(\equiv \dot{q}_{4}\right)$ is a Lagrange multiplier. At this stage the Poisson bracket is defined by

$$
\{A, B\}_{\mathrm{P}} \equiv \frac{\partial A}{\partial q_{\mu}} \frac{\partial B}{\partial \Pi_{\mu}}-\frac{\partial A}{\partial \Pi_{\mu}} \frac{\partial B}{\partial q_{\mu}}, \quad(\mu=1,2,3,4) .
$$

In Eq.(2.29), we use the following notation:

$$
\begin{gathered}
q_{1}=r, \quad q_{2}=\theta, \quad q_{3}=\phi, \\
\Pi_{1}=\Pi_{r}, \quad \Pi_{2}=\Pi_{\theta}, \quad \Pi_{3}=\Pi_{\phi},
\end{gathered}
$$

Requiring the consistency condition on all constraints, we obtain three secondary constraints,

$$
\begin{aligned}
& \dot{\chi}_{1} \approx r-a \equiv \chi_{2} \approx 0, \\
& \dot{\chi}_{2} \approx \frac{\Pi_{r}}{m} \equiv \frac{\chi_{3}}{m} \approx 0, \\
& \dot{\chi}_{3} \approx \frac{1}{m}\left[\frac{\Pi_{\theta}^{2}}{a^{3}}+\frac{\Pi_{\phi}^{2} \sin \theta}{(R+a \sin \theta)^{3}}-m q_{4}\right] \equiv \frac{\chi_{4}}{m} \approx 0, \\
& \dot{\chi}_{4} \approx \frac{\Pi_{\theta} \Pi_{\phi}^{2} \cos \theta}{a(R+a \sin \theta)^{3}}\left(\frac{1}{a}-\frac{\sin \theta}{R+a \sin \theta}\right)-\frac{m^{2}}{3} u \approx 0 .
\end{aligned}
$$


Equation (2.33) determines the Lagrange multiplier $u$; therefore Eq.(2.28) is rewritten as

$$
\begin{aligned}
H= & \frac{1}{2 m}\left[\Pi_{r}^{2}+\frac{\Pi_{\theta}^{2}}{r^{2}}+\frac{\Pi_{\phi}^{2}}{(R+r \sin \theta)^{2}}\right]+q_{4}(r-a) \\
& +\frac{3 \Pi_{\theta} \Pi_{\phi}^{2} \cos \theta}{a m^{2}(R+a \sin \theta)^{3}}\left(\frac{1}{a}-\frac{\sin \theta}{R+a \sin \theta}\right) \chi_{1} .
\end{aligned}
$$

We express $q_{4}$ in term of other variables by Eq.(2.32):

$$
q_{4} \approx \frac{1}{m}\left[\frac{\Pi_{\theta}^{2}}{a^{3}}+\frac{\Pi_{\phi}^{2} \sin \theta}{(R+a \sin \theta)^{3}}\right] .
$$

According to the same argument as in Sec.2.1, the pair $\left(q_{4}, \Pi_{4}\right)$ are taken as dependent variables. Substituting Eq.(2.35) into Eq.(2.34), the Hamiltonian (2.34) is reduced to

$$
\begin{aligned}
H= & \frac{1}{2 m}\left[\Pi_{r}^{2}+\frac{\Pi_{\theta}^{2}}{r^{2}}+\frac{\Pi_{\phi}^{2}}{(R+r \sin \theta)^{2}}\right]+\frac{1}{m}\left[\frac{\Pi_{\theta}^{2}}{a^{3}}+\frac{\Pi_{\phi}^{2} \sin \theta}{(R+a \sin \theta)^{3}}\right](r-a) \\
& +\frac{3 \Pi_{\theta} \Pi_{\phi}^{2} \cos \theta}{m^{2} r(R+r \sin \theta)^{3}}\left(\frac{1}{r}-\frac{\sin \theta}{R+r \sin \theta}\right) \chi_{1} .
\end{aligned}
$$

and here the Poisson bracket is redefined by

$$
\{A, B\}_{\mathrm{P}} \equiv \frac{\partial A}{\partial q_{i}} \frac{\partial B}{\partial \Pi_{i}}-\frac{\partial A}{\partial \Pi_{i}} \frac{\partial B}{\partial q_{i}}, \quad(i=1,2,3) .
$$

Now, we calculate the Dirac brackets of canonical variables $\left(q_{i}, \Pi_{i}, i=1,2,3\right)$ :

$$
\left\{\theta, \Pi_{\theta}\right\}_{\mathrm{D}}=\left\{\phi, \Pi_{\phi}\right\}_{\mathrm{D}}=1
$$

and all other Dirac brackets vanish. From now on, since the system is taken up through the Dirac brackets, we can replace " $\approx$ " by "=". Finally we arrive at

$$
H_{E}=\frac{1}{2 m}\left[\frac{\Pi_{\theta}^{2}}{a^{2}}+\frac{\Pi_{\phi}^{2}}{(R+a \sin \theta)^{2}}\right],
$$

with

$$
r=a, \quad \Pi_{r}=0
$$

Hamilton's equations of motion are given as follows:

$$
\begin{array}{ll}
\dot{r}=0, & \dot{\Pi}_{r}=0, \\
\dot{\theta}=\frac{\Pi_{\theta}}{m a^{2}}, & \dot{\Pi}_{\theta}=-\frac{a \cos \theta \Pi_{\phi}^{2}}{m(R+a \sin \theta)^{3}}, \\
\dot{\phi}=\frac{\Pi_{\phi}}{m(R+a \sin \theta)^{2}}, & \dot{\Pi}_{\phi}=0 .
\end{array}
$$


In the above discussion, we treat $(r, \theta, \phi)$ as coordinate variables. However we can reduce them to $(\theta, \phi)$ by solving the equations $\chi_{2}=0$ and $\chi_{3}=0$ for $r$ and $\Pi_{r}$. In this case, since all constraints are disappeared, we can deal with the system through the Poisson brackets constructed by $\left(\theta, \phi, \Pi_{\theta}, \Pi_{\phi}\right)$. Nevertheless, the resultant Hamiltonian is the same as Eq.(2.39).

\section{Quantum mechanics on a torus}

Now, we consider the quantization of the constrained system discussed in the previous section. In Sec.3.1, we quantize the system in toric coordinates. In Sec.3.2, we bring toric coordinates in after having quantized in Cartesian coordinates.

\subsection{Quantization in toric coordinates}

Following Eq.(2.39), we define the quantum Hamiltonian $\hat{H}$ as:

$$
\hat{H}=\frac{1}{2 m}\left[\frac{\hat{\Pi}_{\theta}^{2}}{a^{2}}+\frac{\hat{\Pi}_{\phi}^{2}}{(R+a \sin \hat{\theta})^{2}}\right],
$$

with

$$
\hat{r}=a, \quad \hat{\Pi}_{r}=0
$$

where we put the notation "^" to stress the operator nature of the affixed. The commutation relations of the canonical operators is

$$
\left[\hat{q}_{m}, \hat{\Pi}_{n}\right]=i \hbar \delta_{m n}, \quad(m, n=2,3)
$$

All other commutators vanish.

According to the representation theory in a general coordinate system developed by De Witt [6], we will rewrite Eq.(3.1) in coordinate representation. We start with a brief review.

On an n-dimensional Riemannian manifold, we suppose to have the commutators of the coordinate operators and their canonical-momentum operators $\left(\hat{x}_{\mu}, \hat{P}_{\mu}\right), \mu=1,2, \ldots, n$ as follows:

$$
\left[\hat{x}^{\mu}, \hat{x}^{\nu}\right]=\left[\hat{P}_{\mu}, \hat{P}_{\nu}\right]=0, \quad\left[\hat{x}^{\mu}, \hat{P}_{\nu}\right]=i \hbar \delta_{\nu}^{\mu}
$$


The wave function of the system is defined by:

$$
\Psi(x, t) \equiv\langle x \mid \Psi\rangle_{t}
$$

where $|x\rangle$ is an eigenvector of the operator $\hat{x}$ and satisfies the orthonormal condition

$$
\left\langle x \mid x^{\prime}\right\rangle=\delta\left(x, x^{\prime}\right)
$$

The generalized delta function $\delta\left(x, x^{\prime}\right)$ means the equations:

$$
\begin{aligned}
\delta\left(x, x^{\prime}\right) & =0, \quad\left(x \neq x^{\prime}\right), \\
\int d \omega f(x) \delta\left(x, x^{\prime}\right) & =f\left(x^{\prime}\right), \quad d \omega \equiv g^{\frac{1}{2}}(x) d^{n} x,
\end{aligned}
$$

with $g(x)$, the determinant of the metric. It is related to Dirac's delta function by:

$$
\delta\left(x, x^{\prime}\right)=g^{\frac{1}{2}}(x) \delta\left(x-x^{\prime}\right)=g^{\frac{1}{2}}\left(x^{\prime}\right) \delta\left(x-x^{\prime}\right) .
$$

From Eq.(3.8) follow two identities:

$$
\begin{aligned}
\left(x^{\mu}-x^{\prime \mu}\right) \frac{\partial}{\partial x^{\nu}} \delta\left(x, x^{\prime}\right) & =-\delta_{\nu}^{\mu} \delta\left(x, x^{\prime}\right) \\
\frac{\partial}{\partial x^{\mu}} \delta\left(x, x^{\prime}\right) & =-\frac{\partial}{\partial x^{\prime \mu}} \delta\left(x, x^{\prime}\right)-\Gamma_{\nu \mu}^{\nu} \delta\left(x, x^{\prime}\right)
\end{aligned}
$$

where $\Gamma_{\nu \lambda}^{\mu}$ is the Cristoffel symbol calculated at $x$ or $x^{\prime}$. With the commutators (3.3), we obtain the equations:

$$
i \hbar \delta_{\nu}^{\mu} \delta\left(x, x^{\prime}\right)=\left(x^{\mu}-x^{\prime \mu}\right)\left\langle x\left|\hat{P}_{\nu}\right| x^{\prime}\right\rangle
$$

and

$$
\left\langle x\left|\hat{P}_{\nu}\right| x^{\prime}\right\rangle=-i \hbar \frac{\partial}{\partial x^{\nu}} \delta\left(x, x^{\prime}\right)+F_{\nu} \delta\left(x, x^{\prime}\right) .
$$

Here $F_{\nu}$ is an arbitrary function of $x$ and $x^{\prime}$.

On account that the momentum operators $\hat{P}_{\mu}$ commute with each other and that $\hat{P}_{\mu}$ should be Hermitian operator, we obtain

$$
\begin{aligned}
F_{\mu} & =\frac{\partial}{\partial x^{\mu}} F \\
F & \equiv R-\frac{1}{2} i \hbar \log g^{\frac{1}{2}}
\end{aligned}
$$


with an arbitrary real function $R$. The function $R$ can be eliminated by unitary transformation:

$$
|x\rangle^{\prime}=\exp \left[-\frac{i}{\hbar} R\right]|x\rangle
$$

Then we have the coordinate representation of $\hat{P}_{\mu}$ :

$$
\left\langle x\left|\hat{P}_{\mu}\right| x^{\prime}\right\rangle=-i \hbar \frac{\partial}{\partial x^{\mu}} \delta\left(x, x^{\prime}\right)-\frac{1}{2} i \hbar \Gamma_{\nu \mu}^{\nu} \delta\left(x, x^{\prime}\right) .
$$

Now having finished a brief overview, we will rewrite the momentum operators $\hat{\Pi}_{\theta}$, $\hat{\Pi}_{\phi}$, using Eq.(3.15), in coordinate representation. The surface element on torus is

$$
d s^{2}=a^{2} d \theta^{2}+(R+a \sin \theta)^{2} d \phi^{2} \equiv g_{m n} d q_{m} d q_{n}, \quad(m, n=2,3) .
$$

It shows that the metric on torus is

$$
\begin{aligned}
g_{m n} & \equiv\left(\begin{array}{cc}
a^{2} & 0 \\
0 & (R+a \sin \theta)^{2}
\end{array}\right), \\
g_{m n} g_{n l}^{-1} & =\delta_{m l} .
\end{aligned}
$$

We obtain the commutation relations of the canonical variables by replacing the Dirac brackets $(\times i \hbar)$ by commutators in Eq.(2.38):

$$
\left[\hat{q}_{m}, \hat{\Pi}_{n}\right]=i \hbar \delta_{m n}, \quad(m, n=2,3)
$$

and all other commutators vanish. Then the momentum operators are expressed as:

$$
\begin{aligned}
\hat{\Pi}_{\theta} & =\frac{\hbar}{i}\left(\frac{\partial}{\partial \theta}+\frac{1}{2} \frac{a \cos \theta}{R+a \sin \theta}\right), \\
\hat{\Pi}_{\phi} & =\frac{\hbar}{i} \frac{\partial}{\partial \phi} .
\end{aligned}
$$

Now, by substituting Eqs.(3.19), (3.20) into Eq.(3.1), we obtain the quantum Hamiltonian $\hat{H}$ :

$$
\begin{aligned}
\hat{H}= & \frac{1}{2 m}\left[\frac{\hat{\Pi}_{\theta}^{2}}{a^{2}}+\frac{\hat{\Pi}_{\phi}^{2}}{(R+a \sin \hat{\theta})^{2}}\right] \\
= & -\frac{\hbar^{2}}{2 m}\left[\frac{1}{a^{2}(R+a \sin \theta)} \frac{\partial}{\partial \theta}\left((R+a \sin \theta) \frac{\partial}{\partial \theta}\right)+\frac{1}{(R+a \sin \theta)^{2}} \frac{\partial^{2}}{\partial \phi^{2}}\right] \\
& +\frac{\hbar^{2}}{8 m} \frac{2 a^{2}+2 a R \sin \theta-a^{2} \cos ^{2} \theta}{a^{2}(R+a \sin \theta)^{2}} .
\end{aligned}
$$


In the last line of Eq.(3.21), a functional term has appeared. It is called the quantum mechanical potential (QMP), having been indicated by De Witt. On the contrary, we alternatively find that we can use the Hamiltonian

$$
\begin{gathered}
\hat{H}=\frac{1}{2 m} \hat{g}^{-\frac{1}{4}} \hat{\Pi}_{m} \hat{g}^{\frac{1}{2}} \hat{g}_{m n}^{-1} \hat{\Pi}_{n} \hat{g}^{-\frac{1}{4}}, \\
\hat{g} \equiv \operatorname{det}\left|\hat{g}_{m n}\right|, \quad(m, n=2,3),
\end{gathered}
$$

instead of the Hamiltonian (3.1). Then the coordinate representation of the Hamiltonian (3.22) is obtained as follows:

$$
\hat{H}=-\frac{\hbar^{2}}{2 m}\left[\frac{1}{a^{2}(R+a \sin \theta)} \frac{\partial}{\partial \theta}\left((R+a \sin \theta) \frac{\partial}{\partial \theta}\right)+\frac{1}{(R+a \sin \theta)^{2}} \frac{\partial^{2}}{\partial \phi^{2}}\right] .
$$

We have no reason to decide which Hamiltonian (3.1) or (3.22) be preferable. This problem of selection of preferable Hamiltonian is well known to appear if one wants to use the polarcoordinate representation in three-dimensional space. There is, however, no theoretical rule for the selection.

\subsection{Quantization in Cartesian coordinates}

In this subsection, we first quantize in the Cartesian coordinate system. We then rewrite in the toric coordinate system, point-transforming the former. The Dirac brackets (2.14) and (2.15) are replaced by commutators $(\times 1 / i \hbar)$

$$
\begin{aligned}
{\left[\hat{x}_{i}, \hat{x}_{j}\right] } & =0 \\
{\left[\hat{x}_{i}, \hat{P}_{j}\right] } & =i \hbar \delta_{i j}-\frac{i \hbar}{a^{2}}\left(\hat{x}_{i}-\frac{\hat{x}_{1} \delta_{1 i}+\hat{x}_{2} \delta_{2 i}}{\sqrt{\hat{x}_{1}^{2}+\hat{x}_{2}^{2}}} R\right)\left(\hat{x}_{j}-\frac{\hat{x}_{1} \delta_{1 j}+\hat{x}_{2} \delta_{2 j}}{\sqrt{\hat{x}_{1}^{2}+\hat{x}_{2}^{2}}} R\right) .
\end{aligned}
$$

In these equations, it is confirmed that $\hat{x}_{i}$ and $\hat{P}_{i}$ are Hermitian operators. Because of the Hermiticity of $\hat{P}_{i}$, the commutators between them obtained from Eq.(2.16) should be

$$
\left[\hat{P}_{i}, \hat{P}_{j}\right]=-\frac{i \hbar}{a^{2}}\left[\left(\hat{x}_{i}-\frac{\hat{x}_{1} \delta_{1 i}+\hat{x}_{2} \delta_{2 i}}{\sqrt{\hat{x}_{1}^{2}+\hat{x}_{2}^{2}}} R\right)\right.
$$




$$
\begin{aligned}
& \times\left(\hat{P}_{j}-\frac{R}{\sqrt{\hat{x}_{1}^{2}+\hat{x}_{2}^{2}}}\left(\hat{P}_{1} \delta_{1 j}+\hat{P}_{2} \delta_{2 j}-\frac{\hat{x}_{1} \delta_{1 j}+\hat{x}_{2} \delta_{2 j}}{\hat{x}_{1}^{2}+\hat{x}_{2}^{2}}\left(\hat{x}_{1} \hat{P}_{1}+\hat{x}_{2} \hat{P}_{2}\right)\right)\right) \\
& -\left(\hat{x}_{j}-\frac{\hat{x}_{1} \delta_{1 j}+\hat{x}_{2} \delta_{2 j}}{\sqrt{\hat{x}_{1}^{2}+\hat{x}_{2}^{2}}} R\right) \\
& \left.\times\left(\hat{P}_{i}-\frac{R}{\sqrt{\hat{x}_{1}^{2}+\hat{x}_{2}^{2}}}\left(\hat{P}_{1} \delta_{1 i}+\hat{P}_{2} \delta_{2 i}-\frac{\hat{x}_{1} \delta_{1 i}+\hat{x}_{2} \delta_{2 i}}{\hat{x}_{1}^{2}+\hat{x}_{2}^{2}}\left(\hat{x}_{1} \hat{P}_{1}+\hat{x}_{2} \hat{P}_{2}\right)\right)\right)\right] .
\end{aligned}
$$

Furthermore, we symmetrize the constraint conditions:

$$
\begin{gathered}
\hat{x}_{3}^{2}-a^{2}+\left(\sqrt{\hat{x}_{1}^{2}+\hat{x}_{2}^{2}}-R\right)^{2}=0 \\
\frac{1}{2}\left(\hat{x}_{i} \hat{P}_{i}+\hat{P}_{i} \hat{x}_{i}\right)-\frac{1}{2} R\left[\frac{1}{\sqrt{\hat{x}_{1}^{2}+\hat{x}_{2}^{2}}}\left(\hat{x}_{1} \hat{P}_{1}+\hat{x}_{2} \hat{P}_{2}\right)+\left(\hat{P}_{1} \hat{x}_{1}+\hat{P}_{2} \hat{x}_{2}\right) \frac{1}{\sqrt{\hat{x}_{1}^{2}+\hat{x}_{2}^{2}}}\right]=0 .
\end{gathered}
$$

Then we define the point transformation of the Cartesian coordinate system to the toric one. The toric coordinate variables defined by Eq.(2.22) are expressed in the Cartesian coordinates as:

$$
\begin{aligned}
& q_{1} \equiv r=x_{3}^{2}+\left(\sqrt{x_{1}^{2}+x_{2}^{2}}-R\right)^{2}, \\
& q_{2} \equiv \theta=\tan ^{-1} \frac{\sqrt{x_{1}^{2}+x_{2}^{2}}-R}{x_{3}} \\
& q_{3} \equiv \phi=\tan ^{-1} \frac{x_{2}}{x_{1}}
\end{aligned}
$$

with

$$
r \sin \theta>-R,
$$

for one-to-one correspondence. At the classical level, the momentum variables in both coordinate systems are related by the point transformation as follows:

$$
\begin{aligned}
\Pi_{i} & =\frac{\partial x_{j}}{\partial q_{i}} P_{j}, \\
P_{i} & =\frac{\partial q_{j}}{\partial x_{i}} \Pi_{j} .
\end{aligned}
$$

The point transformation is available at the quantum level by symmetrizing:

$$
\begin{aligned}
\hat{\Pi}_{i} & =\frac{1}{2}\left[\frac{\partial \hat{x}_{j}}{\partial \hat{q}_{i}} \hat{P}_{j}+\hat{P}_{j} \frac{\partial \hat{x}_{j}}{\partial \hat{q}_{i}}\right], \\
\hat{P}_{i} & =\frac{1}{2}\left[\frac{\partial \hat{q}_{j}}{\partial \hat{x}_{i}} \hat{\Pi}_{j}+\hat{\Pi}_{j} \frac{\partial \hat{q}_{j}}{\partial \hat{x}_{i}}\right] .
\end{aligned}
$$


Substituting Eqs. (3.29) and (3.33) into Eqs. (3.24)-(3.26), we obtain the commutators of the canonical operators in toric coordinates:

$$
\left[\hat{q}_{m}, \hat{\Pi}_{n}\right]=i \hbar \delta_{m n}, \quad(m, n=2,3)
$$

and all other commutators vanish. This equation is just the same as Eq.(3.18), which means that the quantization at the different stage, i.e., at the direct stage of the toric coordinate representation or at the stage of the Cartesian coordinate representation (afterwards point-transforming to the toric coordinate representation), has no influence on the resultant commutators. The constraints (3.27) and (3.28) are rewritten as:

$$
\hat{r}^{2}=a^{2}, \quad \frac{1}{2}\left(\hat{\Pi}_{r} \hat{r}+\hat{r} \hat{\Pi}_{r}\right)=0 .
$$

Next, we will rewrite the quantum Hamiltonian $\hat{H}$ in toric coordinates. The Hamiltonian quantized in Cartesian coordinates is given by

$$
\hat{H}=\frac{1}{2 m} \hat{P}_{i} \hat{P}_{i}
$$

The coordinate transformation in the Hamiltonian is made by substitution of Eqs. (3.29) and (3.33)

The momentum operators $\hat{P}_{i}$ are expressed as:

$$
\begin{aligned}
\hat{P}_{i} & =\left(\hat{\Pi}_{j}\left(\frac{\partial \hat{q}_{j}}{\partial \hat{x}_{i}}\right)+\frac{1}{2}\left[\left(\frac{\partial \hat{q}_{j}}{\partial \hat{x}_{i}}\right), \hat{\Pi}_{j}\right]\right) \\
& =\left(\hat{\Pi}_{j}+\frac{i \hbar}{2}\left(\frac{\partial \hat{x}_{k}}{\partial \hat{q}_{l}}\right)\left(\frac{\partial}{\partial \hat{q}_{j}} \frac{\partial \hat{q}_{l}}{\partial \hat{x}_{k}}\right)\right)\left(\frac{\partial \hat{q}_{j}}{\partial \hat{x}_{i}}\right) \\
& =\hat{g}^{-\frac{1}{4}} \hat{\Pi}_{j} \hat{g}^{\frac{1}{4}}\left(\frac{\hat{q}_{j}}{\hat{x}_{i}}\right), \quad(i, j, k=1,2,3) .
\end{aligned}
$$

In the process from the second line to the third of Eq.(3.37), we have made use of the matrix identity

$$
\operatorname{tr}\left[M^{-1} \partial_{k} M\right]=(\operatorname{det} M)^{-1} \partial_{k} \operatorname{det} M
$$

Now we introduce new momentum operators $\hat{P}_{i}{ }_{i}$, which are defined as the Cartesian momentum operators restricted to the constraint condition (3.35). They are expressed as:

$$
\hat{P}_{i}^{\prime}=\sum_{m=2}^{3} \hat{g}^{-\frac{1}{4}} \hat{\Pi}_{m} \hat{g}^{\frac{1}{4}}\left(\frac{\partial \hat{q}_{m}}{\partial \hat{x}_{i}}\right),
$$


and interpreted as the projected Cartesian momentum operators onto the torus, and should be observable.

We will check the Hermiticity of $\hat{P}_{3}^{\prime}$. By Eq.(3.39), $\hat{P}_{3}^{\prime}$ is

$$
\begin{aligned}
\hat{P}_{3}^{\prime} & =-\frac{1}{a} \sin \theta \hat{\Pi}_{\theta}-\frac{3 \hbar}{2 i a} \cos \theta \\
& =-\frac{\hbar}{i}\left[\frac{1}{a} \sin \theta \frac{\partial}{\partial \theta}+\frac{1}{a} \cos \theta+\frac{\sin \theta \cos \theta}{R+a \sin \theta}\right] .
\end{aligned}
$$

If we substitute $\Pi_{r}=0$ into Eq.(3.33), we have

$$
\begin{aligned}
\hat{P}_{3}^{\prime \prime} & =\frac{1}{2} \sum_{m=2}^{3}\left[\frac{\partial \hat{q}_{m}}{\partial \hat{x}_{3}} \hat{\Pi}_{m}+\hat{\Pi}_{m} \frac{\partial \hat{q}_{m}}{\partial \hat{x}_{3}}\right] \\
& =-\frac{\hbar}{i}\left[\frac{1}{a} \sin \theta \frac{\partial}{\partial \theta}+\frac{1}{2 a} \cos \theta+\frac{1}{2} \frac{\sin \theta \cos \theta}{R+a \sin \theta}\right] .
\end{aligned}
$$

The result does not coincide with the above equation (3.40). In Eqs.(3.40) and (3.41), we find that $P^{\prime \prime}{ }_{3}$ is a Hermitian operator, while $P^{\prime}{ }_{3}$ is not. This teaches us to put the restriction $\hat{\Pi}_{r}=0$ on the Hamiltonian after the substitution of Eq.(3.37) into Eq.(3.36). We arrive at the resultant Hamiltonian

$$
\begin{aligned}
\hat{H} & =\frac{1}{2 m} \hat{P}_{i} \hat{P}_{i}^{\dagger} \\
& =\frac{1}{2 m} \hat{g}^{-\frac{1}{4}} \hat{\Pi}_{m} \hat{g}^{\frac{1}{2}} \hat{g}_{m n}^{-1} \hat{\Pi}_{n} \hat{g}^{-\frac{1}{4}}
\end{aligned}
$$

The Hamiltonian (3.42) has no QMP in contrast to Eq.(3.22), and is a Hermitian operator. We finally obtain the coordinates representation of the Hamiltonian, whose system has first been quantized in Cartesian coordinates and point-transformed into toric coordinates afterward. Namely we thus have

$$
\hat{H}=-\frac{\hbar^{2}}{2 m}\left[\frac{1}{a^{2}(R+a \sin \theta)} \frac{\partial}{\partial \theta}\left((R+a \sin \theta) \frac{\partial}{\partial \theta}\right)+\frac{1}{(R+a \sin \theta)^{2}} \frac{\partial^{2}}{\partial \phi^{2}}\right] .
$$

\section{Conclusion}

We have quantized our constrained system based on the Dirac formalism for the classical constrained system as well as on the canonical-quantization method. A preferable quantum Hamiltonian for a particle constrained on a torus has been obtained. 
In quantum theory, to express the momentum operators in coordinate representation and to have the Schrödinger equation, the commutators of canonical variables play an essential role. The commutators (3.24)-(3.26) in the Cartesian coordinate system are much complicated. Therefore, it is very difficult to obtain the coordinate representation of the momentum operators in this system. Using the toric coordinate system enables us to have simple commutators and represent the momentum operators as operators in coordinate space. Although the toric coordinate system defined by Eq. (2.22) has a restriction $r \sin \theta>-R$, it is adequate to deal with the constrained system of a particle on the torus. With this coordinate system we have two Hamiltonians Eqs.(3.21) and (3.23), the former with QMP and the latter without QMP. With which Hamiltonian (3.1) or (3.22) we start, in momentum representation, gives rise to this difference.

In quantum theory, the order that we first make a coordinate transformation then restrict the system under some constraint conditions, or vice versa, gives a great influence on the resultant

theory. We conclude, in this paper, that, under the condition $\hat{\Pi}_{r}=0$, the point transformations (3.32) and (3.33) are quite misleading. We must first transform the Hamiltonian into the form shown in Eq.(3.42), then afterwards restrict it to the constraint condition $\hat{\Pi}_{r}=0$. We thus have a preferable quantum Hamiltonian Eq.(3.43).

\section{Acknowledgement}

Two of the authors (K.Y. and M.Y.) would like to thank Iwanami Fûjukai for financial support. 


\section{References}

[1] P. A. M. Dirac, Lectures on Quantum Mechanics (Yeshiva Univ. Press, New York, 1964); K. Sundermeyer, Constrainted Dynamics, Lecture Note in Physics vol.169 (Springer, Berlin, 1982).

[2] For $S^{D}$ : Y. Ohnuki and S. Kitakado, J.Math.Phys.34 (1993) 2827.

[3] N. K. Falck and A. C. Hirshfeld, Eur.J.Phys.4 (1983) 5.

[4] W. S. Massey, A Basic Course in Algebraic Topology (Springer, Berlin, 1991).

[5] T. Homma, T. Inamoto and T.Miyazaki, Phys.Rev.D 42 (1990) 2049.

[6] B. S. DeWitt, Phys.Rev.85 (1952) 653. 


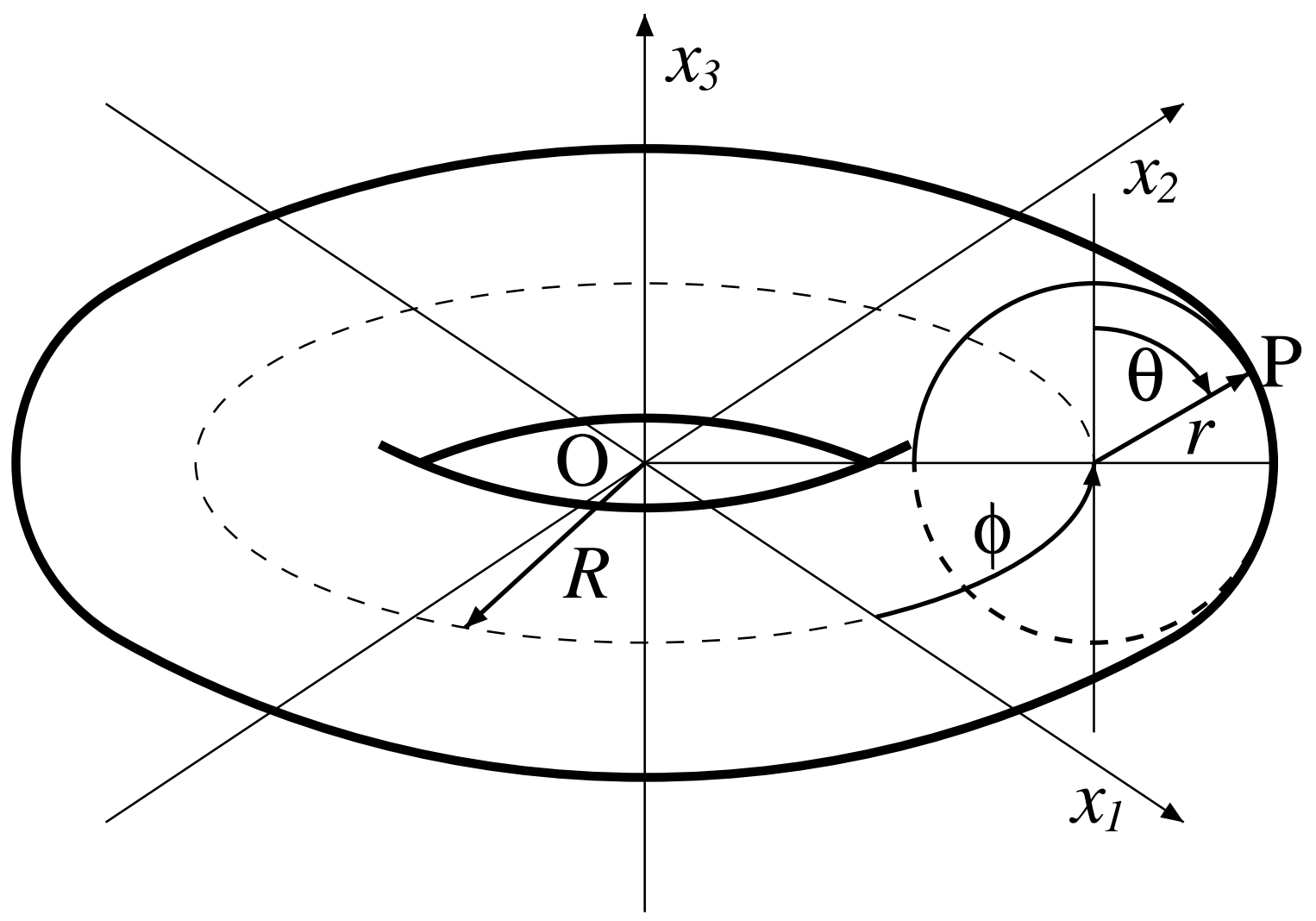

Fig. 1. Toric coordinate system. 